\section{Investigación original}

dx.doi.org/10.35366/CMA201E

\title{
Fentanyl-bupivacaína y bupivacaína en intervenciones quirúrgicas
}

\section{Fentanyl-bupivacaine and bupivacaine in surgical procedures}

\author{
Dr. Karell Piñón-García, ${ }^{*} \ddagger$ Dra. Mirelys Valladares-Díaz, \\ Dra. Mayda Correa-Borrell, ${ }^{\S}$ Dr. José Antonio Pozo-Romero," \\ Dr. Carlos de la Paz-Estradaq
}

RESUMEN. Objetivos: Evaluar las modalidades analgésicas subaracnoideas fentanyl-bupivacaína y bupivacaína en intervenciones quirúrgicas del hemiabdomen inferior. Material y métodos: Se realizó un estudio prospectivo longitudinal analítico en el Hospital Universitario «Manuel Ascunce Domenech» desde enero de 2015 a diciembre de 2017. El universo estuvo compuesto por 180 pacientes. Se seleccionó una muestra de 60 pacientes mediante muestreo probabilístico aleatorio simple distribuidos en dos grupos: A (fentanyl-bupivacaína) y B (bupivacaína) que cumplieron los criterios de inclusión. Los datos fueron recogidos en formulario a propósito de la investigación que permitió la confección de una base de datos en Statistical Package for Social Sciences. Se emplearon en el análisis técnicas univariadas y multivariadas. Resultados: Las complicaciones y reacciones adversas y su probabilidad de presentación en el grupo fentanyl-bupivacaína, que resultó mayor, fueron somnolencia (3.5 veces), hipotensión arterial (2.8 veces), prurito (2.1 veces) y bradicardia (2.1 veces), corroborado por el intervalo de confianza. Este grupo tuvo 10 veces más probabilidades de tener molestia tolerable o nada de dolor $(O R=10.2)$ que los tratados con bupivacaína que presentaron dolor moderado; 10 veces más probabilidades de analgesia superior a tres horas $(O R=10.7)$ frente a bupivacaína con analgesia de treinta minutos a tres horas y ocho veces más probabilidades de percibir satisfacción buena $(\mathrm{OR}=7.8)$. Conclusiones: La evaluación del dolor postoperatorio y el tiempo de analgesia fueron mejores en los pacientes tratados con fentanyl-bupivacaína, lo que resultó en analgesia de mayor calidad aunque predominó la somnolencia, la hipotensión arterial, el prurito y la bradicardia como reacciones adversas.

ABSTRACT. Objective: To evaluate subarachnoid analgesic modes fentanyl-bupivacaine and bupivacaine in surgery procedures of low abdomen. Material and methods: An analytic longitudinal prospective study was performed at the «Manuel Ascunce Domenech» University Hospital in Camagüey, from January 2015 to December 2017. The universe was compound by 180 patients. Sample was selected about 60 patients by probabilistic simple random sampling distributed in two groups: A (fentanyl-bupivacaine) and B (bupivacaine) according to inclusion criteria. The data were collected in questionnaire properly to investigation with baseline data in Statistical Package for Social Sciences. A univariate and multivariate analysis were conducted. Results: Complications and adverse reactions and their probability of presentation in the fentanyl-bupivacaine group was greater, drowsiness (3.5 times), arterial hypotension (2.8 times), pruritus (2.1 times) and bradycardia (2.1 times) corroborated by confidence intervals. This group had ten times more probability to have tolerable trouble or no pain $(O R=10.2)$ that bupivacaine group, all of which showed moderate pain; 10 times more probability of analgesia higher to 3 hours $(O R=10.7)$ versus bupivacaine with analgesia from 30 minutes to 3 hours and 8 times more probability to perceive good satisfaction $(O R=7.8)$. Conclusions: Evaluation of pain postoperative and analgesic time were better in patients managed with fentanylbupivacaine that resulted in analgesic of the greatest quality although prevailed drowsiness, arterial hypotension, pruritus and bradycardia like adverse reactions.
Anestesiología

Enero-Marzo 2020

Vol. 43. No. 1. pp 29-33

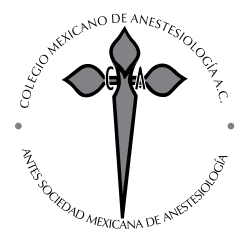

${ }^{*}$ Profesor Instructor.

‡ Especialista de primer grado en Anestesiología y Reanimación. Hospital Universitario «Manuel Ascunce Domenech». Camagüey, Cuba.

$\S$ Especialista de segundo grado en Anestesiología y Reanimación. Máster en Urgencias Médicas. Investigadora auxiliar. Profesor Auxiliar y Consultante. Hospital Universitario «Manuel Ascunce Domenech». Camagüey, Cuba. \| Especialista de segundo grado en Anestesiología y Reanimación. Máster en Urgencias Médicas. Profesor asistente. Hospital Universitario «Manuel Ascunce Domenech». Camagüey, Cuba.

" Especialista de segundo grado en Anestesiología y Reanimación. Máster en Urgencias Médicas. Profesor asistente. Hospital Pediátrico Provincial «Ignacio Agramonte Piña». Camagüey, Cuba.

Palabras clave: Dolor postoperatorio, analgesia, satisfacción, reacciones adversas.

Keywords: Pain postoperative, analgesia, satisfaction, adverse reactions.

Solicitud de sobretiros:

Dr. Carlos de la Paz-Estrada

Edificio 34 Apto. 30 E, Calle 6ta. y Mónaco Este, Rpto. Julio Antonio Mella, Zona Postal Camagüey 6, 70600, Camagüey, Cuba. E-mail: carlosdelapazestrada@gmail.com cdelapaz.cmw@infomed.sld.cu

Recibido para publicación: 15-08-2018

Aceptado para publicación: 07-08-2019 


\section{INTRODUCCIÓN}

$\mathrm{E}^{1}$ l dolor postoperatorio considerado también un signo vital clave, es una variante del dolor agudo; es uno de los peores tratados, pudiendo durar horas o días, produce ansiedad y angustia. Es producido por una hiperestimulación de las vías nociceptivas.

El control del dolor en el postoperatorio indica la calidad de la atención sanitaria que se le brinda a un paciente. Se requiere de la utilización de estrategias para valorar su intensidad y evaluar la calidad del tratamiento recibido ${ }^{(1,2)}$ y los protocolos para su tratamiento avanzan de manera significativa en la actualidad. Sin embargo, se reporta que la prevalencia de dolor moderado a severo después de las intervenciones quirúrgicas se encuentra alrededor de 26 a 33\% y de dolor severo entre 8 y $13 \%{ }^{(3)}$.

El inadecuado control del dolor conduce a un fenómeno nociceptivo persistente e invalidante que ocasiona sufrimiento e insatisfacción en los pacientes y trae como consecuencia complicaciones. El adecuado tratamiento se considera un indicador relevante de buena práctica clínica y de una elevada calidad asistencial. La evidencia actual demuestra que el correcto control del dolor agudo postoperatorio facilita la recuperación, lo cual reduce el tiempo de estancia hospitalaria, los costos y además disminuye la morbilidad y mortalidad asociada al cuidado en un centro asistencial ${ }^{(4,5)}$. Por ello, la disminución del dolor postoperatorio es la piedra angular de una evolución adecuada.

Existe una amplia variedad de medicamentos útiles para el tratamiento y control del dolor en el postoperatorio. La recomendación más práctica es la asociación de dos analgésicos con mecanismos de acción distintos, con lo cual se consigue una analgesia mayor y además se reducen los efectos indeseables asociados con las dosis de algunos de los fármacos ${ }^{(6,7)}$. La combinación de fentanyl (potente analgésico narcótico de acción inmediata y vida media corta) y bupivacaína (anestésico local con latencia más larga, de 20 minutos, y duración más prolongada entre 3 y 9 horas) ${ }^{(8,9)}$, aprovecha las cualidades analgésicas de cada uno, ideal para el transoperatorio el primero y garantía de analgesia en el postoperatorio el segundo. Los opiáceos son efectivos para el tratamiento del dolor visceral y los anestésicos locales para el alivio del dolor somático. La bupivacaína al combinarla con fentanyl a bajas dosis brinda una analgesia de 60 a 90 minutos y a su vez disminuye el período de latencia de la bupivacaína con menor frecuencia de reacciones adversas ${ }^{(10,11)}$.

No obstante, una gran parte de estos pacientes siguen tratados de forma inadecuada, por lo que experimentan un sufrimiento injustificado que incrementa el riesgo de complicaciones postoperatorias ${ }^{(12-14)}$, lo cual motiva a la realización de este estudio, que pretende determinar en dos grupos las complicaciones postoperatorias, la evaluación del dolor postoperatorio, el tiempo de duración de la analgesia y la satisfacción percibida por el paciente con la analgesia empleada durante el postoperatorio para lograr confort y disminuir las complicaciones en los pacientes quirúrgicos.

\section{MATERIAL Y MÉTODOS}

La investigación constituyó un estudio prospectivo, longitudinal y analítico, con el objetivo de evaluar las modalidades analgésicas subaracnoideas fentanyl-bupivacaína y bupivacaína en intervenciones quirúrgicas del hemiabdomen inferior en el Hospital Universitario «Manuel Ascunce Domenech» desde enero de 2015 hasta diciembre de 2017. El universo estuvo compuesto por 180 pacientes. Se seleccionó mediante muestreo probabilístico aleatorio simple una muestra de 60 pacientes distribuidos en dos grupos (A y B) con modalidades terapéuticas diferentes y que cumplieron los criterios de inclusión. La pertenencia al grupo fue determinada de forma aleatoria. El tamaño muestral fue calculado con un nivel de seguridad de 95\%, precisión de 3\%, proporción de 5\% y proporción esperada de pérdidas de $15 \%$.

Criterios de inclusión:

- Edad entre 18 y 55 años.

- Clasificación I y II según la American Society of Anesthesiologists.

Criterio de exclusión:

- Contraindicaciones para anestesia espinal.

Las modalidades terapéuticas fueron:

- Grupo A: bupivacaína $0.5 \% 12.5 \mathrm{mg}$ más fentanyl $25 \mu \mathrm{g}$.

- Grupo B: bupivacaína $0.5 \% 15 \mathrm{mg}$.

Se aplicaron técnicas estadísticas de contraste de proporciones mediante $\chi^{2}$ con un nivel de confiabilidad del $95 \%$. Se empleó la determinación de odds-ratio, intervalo de confianza y significación estadística.

\section{RESULTADOS}

La Tabla 1 expone las complicaciones y reacciones adversas encontradas por grupos, así como la probabilidad de presentación de ellas. De esta manera se observó que es 3.5 veces más probable que el paciente del grupo A (al que se le administró fentanyl-bupivacaína) tenga somnolencia en relación a los pacientes del grupo B (a los que se les administró bupivacaína), esto corroborado por el intervalo de confianza 2.287-5.687. Es 2.8 veces más probable que el paciente del grupo A tenga 


\begin{tabular}{|c|c|c|c|c|c|c|c|c|c|}
\hline \multirow[b]{2}{*}{ Complicaciones } & \multicolumn{2}{|c|}{ Grupo A } & \multicolumn{2}{|c|}{ Grupo B } & \multicolumn{2}{|c|}{ Total } & \multirow[b]{2}{*}{ OR $(A / B)$} & \multirow[b]{2}{*}{ IC 95\% } & \multirow[b]{2}{*}{ Significancia } \\
\hline & $\mathrm{n}$ & $\%$ & $\mathrm{n}$ & $\%$ & $\mathrm{n}$ & $\%$ & & & \\
\hline Somnolencia & 6 & 10.0 & 2 & 3.3 & 8 & 13.3 & 3.500 & $2.287-5.687$ & $8.50 \mathrm{E}-10$ \\
\hline Náuseas y vómitos & 2 & 3.3 & 5 & 8.3 & 7 & 11.7 & 0.357 & $0.124-1.324$ & 2.36E-01 \\
\hline Hipotensión & 5 & 8.3 & 2 & 3.3 & 7 & 11.7 & 2.800 & $2.275-4.395$ & $2.36 \mathrm{E}-10$ \\
\hline Prurito & 4 & 6.7 & 2 & 3.3 & 6 & 10.0 & 2.154 & $2.745-5.245$ & $6.19 E-11$ \\
\hline Bradicardia & 4 & 6.7 & 2 & 3.3 & 6 & 10.0 & 2.154 & $2.895-7.271$ & $6.19 \mathrm{E}-11$ \\
\hline Hipertensión & 1 & 1.7 & 2 & 3.3 & 3 & 5.0 & 0.483 & $0.247-1.112$ & 8.44E-02 \\
\hline
\end{tabular}

hipotensión arterial (corroborado por el intervalo de confianza 2.275-4.395). Es 2.1 veces más probable que los del grupo A tengan prurito (intervalo de confianza 2.745-5.245). Es 2.1 veces más probable que el paciente del grupo A tenga bradicardia en relación a los pacientes del grupo B (intervalo de confianza 2.895-7.271).

Los sujetos tratados con fentanyl-bupivacaína tuvieron aproximadamente 10 veces más probabilidades de tener molestia tolerable o nada de dolor que los tratados con bupivacaína $(\mathrm{OR}=10.2)$ que refirieron dolor moderado como muestra la Tabla 2.

La Tabla 3 muestra que aquéllos tratados con fentanylbupivacaína tuvieron 10 veces más probabilidades de tener analgesia de más de tres horas que los tratados con bupivacaína $(\mathrm{OR}=10.7)$ que tuvieron analgesia de 30 minutos a tres horas.

En la Tabla 4 se demuestra que los pacientes tratados con fentanyl-bupivacaína tuvieron aproximadamente ocho veces más probabilidades de tener satisfacción percibida buena que los tratados con bupivacaína (OR = 7.8).

\section{DISCUSIÓN}

Las complicaciones y reacciones adversas en la presente investigación coinciden con el reporte de Fernández Ramos y colaboradores ${ }^{(15)}$, en su estudio sobre el fentanyl intradural en ancianos señalan que la somnolencia y la bradicardia se encontró en 55 y 46 pacientes, respectivamente, y en varios de ellos se observó prurito e hipotensión. Estos autores mencionan que en ningún paciente se presentaron complicaciones graves como la depresión respiratoria y la retención de orina, y los efectos se produjeron en la medida en que se incrementó la dosis del opiáceo. Asimismo, reportaron que no existió prurito en el grupo de pacientes en los que no se empleó fentanyl; sin embargo, casi la totalidad en los que se aplicó dosis altas del mismo mostraron este efecto.

A su vez, existe correspondencia con otros autores. Miyoshi y su grupo ${ }^{(16)}$ reportan las complicaciones y reacciones ad- versas en su serie de pacientes tratados con fentanyl: náuseas (25.3\%), somnolencia (41.7\%) y constipación (8.3\%).

Singla y colegas ${ }^{(17)}$ señalan que en el tratamiento con fentanyl del dolor por encima de cuatro en la EVA, la somnolencia puede aparecer de $30-50 \%$. Kang y su equi$\mathrm{po}^{(18)}$, reportan que la somnolencia puede ser prevenida mediante la disminución de las dosis de medicamento. Unlü ${ }^{(19)}$ demuestra en su investigación que el tratamiento con opiáceos no afecta el proceso cognitivo a pesar de que puede ocasionar somnolencia en algunos pacientes. Li y colaboradores ${ }^{(20)}$ reportan pocas reacciones adversas en su serie de pacientes tratados con la combinación de fentanyl y bupivacaína.

González Pérez y asociados(21) reportan un estudio comparativo entre bupivacaína y bupivacaína-fentanyl en cirugía por fractura de cadera en ancianos y obtiene mayor estabilidad hemodinámica en el grupo del fentanyl con un mayor número de episodios hipotensivos en el de la bupivacaína, sin coincidir con nuestros resultados en los que el grupo fentanyl-bupivacaína presentó mayor probabilidad de hipotensión arterial y bradicardia, ya que la mayoría de los opioides disminuyen el tono simpático y aumentan el tono vagal ${ }^{(22)}$.

\begin{tabular}{|c|c|c|c|c|c|c|}
\hline \multirow[b]{2}{*}{ Evaluación del dolor } & \multicolumn{2}{|c|}{ Grupo A } & \multicolumn{2}{|c|}{ Grupo B } & \multicolumn{2}{|c|}{ Total } \\
\hline & $\mathrm{n}$ & $\%$ & $\mathrm{n}$ & $\%$ & $\mathrm{n}$ & $\%$ \\
\hline Nada de dolor & 6 & 10.0 & 2 & 3.3 & 8 & 13.3 \\
\hline Molestia tolerable & 21 & 35.0 & 12 & 20.0 & 33 & 55.0 \\
\hline Dolor moderado & 2 & 3.3 & 14 & 23.3 & 16 & 26.7 \\
\hline Dolor intenso & 1 & 1.7 & 2 & 3.3 & 3 & 5.0 \\
\hline Total & 30 & 50.0 & 30 & 50.0 & 60 & 100 \\
\hline
\end{tabular}




\begin{tabular}{|c|c|c|c|c|c|c|}
\hline \multirow[b]{3}{*}{ Tiempo de analgesia } & $\begin{array}{l}\text { a 3: } \\
\text { n de }\end{array}$ & $\begin{array}{l}\text { iempc } \\
\text { a ana }\end{array}$ & esi: & & & \\
\hline & \multicolumn{2}{|c|}{ Grupo A } & \multicolumn{2}{|c|}{ Grupo B } & \multicolumn{2}{|c|}{ Total } \\
\hline & $\mathrm{n}$ & $\%$ & $\mathrm{n}$ & $\%$ & $\mathrm{n}$ & $\%$ \\
\hline Menos de 30 minutos & 2 & 3.3 & 3 & 5.0 & 5 & 8.3 \\
\hline 30 minutos-3 horas & 5 & 8.3 & 16 & 26.7 & 21 & 35.0 \\
\hline Más de tres horas & 23 & 38.3 & 7 & 11.7 & 30 & 50.0 \\
\hline Total & 30 & 50.0 & 26 & 43.3 & 56 & 93.0 \\
\hline
\end{tabular}

Parte del efecto analgésico de los opiáceos es realizado a través de la activación de neuronas serotoninérgicas. Se ha observado que el fentanilo origina una regulación a la alta dosis dependiente de los receptores 5- $\mathrm{HT}_{1 \mathrm{~A}}$ a nivel del hipocampo y de la amígdala de cerebro de rata. Se señala en la literatura que la estimulación de los receptores 5- $\mathrm{HT}_{1 \mathrm{~A}}$ presenta efecto analgésico a nivel central ${ }^{(23-25)}$. Estos planteamientos concuerdan con nuestros resultados en los que los sujetos con asociación fentanyl-bupivacaína presentaron menor dolor postoperatorio y mayor tiempo de analgesia.

La asociación de mayor tiempo y calidad de analgesia postoperatoria redunda en una mayor satisfacción subjetiva por parte del paciente, que se siente beneficiado con la aplica-

\begin{tabular}{|c|c|c|c|c|c|c|}
\hline \multicolumn{7}{|c|}{$\begin{array}{l}\text { Tabla 4: Satisfacción percibida con } \\
\text { la analgesia postoperatoria. }\end{array}$} \\
\hline \multirow[b]{2}{*}{ Satisfacción percibida } & \multicolumn{2}{|c|}{ Grupo A } & \multicolumn{2}{|c|}{ Grupo B } & \multicolumn{2}{|c|}{ Total } \\
\hline & $\mathrm{n}$ & $\%$ & $\mathrm{n}$ & $\%$ & $\mathrm{n}$ & $\%$ \\
\hline Buena & 27 & 45.0 & 16 & 26.7 & 43 & 71.7 \\
\hline Regular & 2 & 3.3 & 12 & 20.0 & 14 & 23.3 \\
\hline Mala & 1 & 1.7 & 2 & 3.3 & 3 & 5.0 \\
\hline Total & 30 & 50.0 & 30 & 50.0 & 60 & 100 \\
\hline
\end{tabular}

ción de esquemas terapéuticos cada vez más efectivos, como quedó demostrado en el estudio, coincidente con Hefni y su grupo $^{(26)}$, Graudins y colegas ${ }^{(27)}$ y Adamou y colaboradores ${ }^{(28)}$ que señalan satisfacción subjetiva en pacientes tratados con opioides para el control del dolor.

Concluimos que la asociación de fentanyl-bupivacaína resultó en analgesia de mayor calidad que la bupivacaína en las intervenciones de hemiabdomen inferior con mejores resultados en relación a tiempo de analgesia y evaluación del dolor postoperatorio; así como la presencia de complicaciones y reacciones adversas fueron mayores en el grupo fentanylbupivacaína con predominio de somnolencia, hipotensión arterial, prurito y bradicardia.

\section{REFERENCIAS}

1. White PF, Elvir-Lazo OL, Zaentz AS, Kariger R, Yumul R, Khany MM, et al. Does small-dose fentanyl improve perioperative outcomes in the ambulatory setting? A randomized, double-blind, placebo-controlled study. Acta Anaesthesiol Scand. 2015;59:56-64.

2. Takigawa C, Goto F, Tanda S, shima Y, Yomiya K, Matoba M, et al. Breakthrough pain management using fentanyl buccal tablet (fbt) in combination with around-the-clock (atc) opioids based on the efficacy and safety of fbt, and its relationship with atc opioids: results from an open-label, multi-center study in japanese cancer patients with detailed evaluation. J Clin Oncol. 2015;45:67-74.

3. Yoshida K, Nishizawa D, Ichinomiya T, Ichinohe T, Hayashida M, Fukuda K, et al. Prediction formulas for individual opioid analgesic requirements based on genetic polymorphism analyses. PLoS One. 2015;10:e0116885.

4. Yang CH, Tian X, Yin HB, Gao XH, Li N. Sedation and analgesia with fentanyl and etomidate for intrathecal injection in childhood leukemia patients. Medicine (Baltimore). 2015;94:e361.

5. Xing SZ, Zhang Y. Efficacy and safety of transdermal fentanyl for the treatment of oral mucositis pain caused by chemoradiotherapy in patients with esophageal squamous cell carcinoma. Support Care Cancer. 2015;23:753-759.

6. Sviggum HP, Yacoubian S, Liu X, Tsen LC. The effect of bupivacaine with fentanyl temperature on initiation and maintenance of labor epidural analgesia: a randomized controlled study. Int J Obstet Anesth. 2015;24:15-21.

7. Robleda G, Roche-Campo F, Urrutia G, Navarro M, Sendra MA, Castillo $\mathrm{A}$, et al. A randomized controlled trial of fentanyl in the pre-emptive treatment of pain associated with turning in patients under mechanical ventilation: research protocol. J Adv Nurs. 2015;71:441-450.

8. Craig MG, Grant EN, Tao W, McIntire DD, Leveno KJ. A randomized control trial of bupivacaine and fentanyl versus fentanyl-only for epidural analgesia during the second stage of labor. Anesthesiology. 2015;122:172-177.

9. Chen CC, Yang CC, Hu CC, Shih HN, Chang YH, Hsieh PH. Acupuncture for pain relief after total knee arthroplasty: a randomized controlled trial. Reg Anesth Pain Med. 2015;40:31-36.

10. Bouattour K, Moyano-Tidou G, Le Gouez A, Martel-Jacob S, Mercier FJ. Epidural bleeding after labor epidural analgesia. Ann Fr Anesth Reanim. 2014;33:690-692.

11. Júnior Ade P, Erdmann TR, Santos TV, Brunharo GM, Filho CT, Losso MJ, et al. Comparison between continuous thoracic epidural and paravertebral blocks for postoperative analgesia in patients undergoing thoracotomy: Systematic review. Braz J Anesthesiol. 2013;63:433-442.

12. Holler JP, Ahlbrandt J, Burkhardt E, Gruss M, Rohrig R, Knapheide J, et al. Peridural analgesia may affect long-term survival in patients with colorectal cancer after surgery (PACO-RAS-Study): an analysis of a cancer registry. Ann Surg. 2013;258:989-993.

13. Erkin Y, Aydin Z, Tasdogen A, Karci A. A new technique for long time catheterization of sacral epidural canal in rabbits. Rev Bras Anestesiol. 2013;63:385-388.

14. Di Minno MN, Milone M, Russolillo A, Lupoli R, Di Minno A, Maietta $\mathrm{P}$, et al. Ropivacaine infusion in diabetics subject with peripheral arterial disease. A prospective study. Exp. Clin Endocrinol Diabetes. 2013;121:91-93. 
15. Fernández-Ramos H, Pozo-Romero JA, Correa-Borrel M. Comportamiento de la dosis intradural de fentanil en el paciente geriátrico. Rev Cuba Anestesiol Reanim. 2009;8:726-732.

16. Miyoshi T, Yamauchi H, Misumi N, Goto T, Kai S, Yoshida E, et al. Efficacy and safety of transdermal fentanyl patches for opioid initiation in patients with gastrointestinal obstruction. Gan To Kagaku Ryoho. 2014;41:1401-1405.

17. Singla NK, Muse DD, Evashenk MA, Palmer PP. A dose-finding study of sufentanil sublingual microtablets for the management of postoperative bunionectomy pain. J Trauma Acute Care Surg. 2014;77:S198-S203.

18. Kang JH, Oh SY, Song SY, Lee HY, Kim JH, Lee KE, et al. The efficacy of low-dose transdermal fentanyl in opioid-naive cancer patients with moderate-to-severe pain. Korean J Intern Med. 2015;30:88-95.

19. Unlü N, Bilen A, Ali A, Turgut N, Türkmen A. The cognitive effects of using transdermal fentanyl in cancer pain. Agri. 2014;26:126-130.

20. Li B, Wang H, Gao C. Bupivacaine in combination with fentanyl or sufentanil in epidural/intrathecal analgesia for labor: a meta-analysis. J Clin Pharmacol. 2015;55:584-591.

21. González-Pérez E, Águila-Soto PC, Pérez-Sainz RA, Nieto-Monteagudo CG, González-Cabrera N, Ramírez-Méndez M. Calidad en la anestesia espinal con bupivacaína más fentanyl para pacientes con fractura intertrocantérica de cadera. Rev Cuba Anestesiol Reanim. 2009;8:128-147.

22. Navarrete-Zuazo VM. Anestesia intravenosa. En: Dávila Cabo de Villa E. Anestesiología clínica. La Habana: Ciencias Médicas; 2014. pp. 99-138.
23. Timerbaev VH, Smimova OV, Genov PG, Olejnikova ON, Rebrova OY. Assessment of pain relief in patients receiving different variants of multimodal analgesia after major gynecological surgery. Anesteziol Reanimatol. 2014;(2):32-37.

24. Barann M, Stamer UM, Lyutenska M, Stuber F, Bonisch H, Urban B. Effects of opioids on human serotonin transporters. Naunyn Schmiedebergs Arch. Pharmacol. 2015;388:43-49.

25. Hillman AD, Witenko CJ, Sultan SM, Gala G. Serotonin syndrome caused by fentanyl and methadone in a burn injury. Pharmacotherapy. 2015;35:112-117.

26. Hefni AF, Mahmoud MS, Al Alim AA. Epidural dexamethasone for postoperative analgesia in patients undergoing abdominal hysterectomy: A dose ranging and safety evaluation study. Saudi J Anaesth. 2014;8:323327.

27. Graudins A, Meek R, Egerton-Warburton D, Oakley E, Seith R. The PICHFORK (Pain in Children Fentanyl or Ketamine) trial: a randomized controlled trial comparing intranasal ketamine and fentanyl for the relief of moderate to severe pain in children with limb injuries. Ann Emerg Med. 2015;65:248-254.e1.

28. Adamou N, Tukur J, Muhammad Z, Galadanci H. A randomised controlled trial of opioid only versus combined opioid and non-steroidal anti inflammatory analgesics for pain relief in the first 48 hours after Caesarean section. Niger Med J. 2014;55:369-373. 